\title{
Dynamics of outer mitochondrial membrane permeabilization during apoptosis
}

\author{
M Rehm ${ }^{\star, 1,2}$, HJ Huber ${ }^{1,2,3}$, CT Hellwig ${ }^{1}$, S Anguissola ${ }^{1}$, H Dussmann ${ }^{1}$ and JHM Prehn" ${ }^{\star, 1,2}$
}

Individual cells within a population undergo apoptosis at distinct, apparently random time points. By analyzing cellular mitotic history, we identified that sibling HeLa cell pairs, in contrast to random cell pairs, underwent apoptosis synchronously. This allowed us to use high-speed cellular imaging to investigate mitochondrial outer membrane permeabilization (MOMP), a highly coordinated, rapid process during apoptosis, at a temporal resolution approximately 100 times higher than possible previously. We obtained new functional and mechanistic insight into the process of MOMP: We were able to determine the kinetics of pore formation in the outer mitochondrial membrane from the initiation phase of cytochrome-c-GFP redistribution, and showed differential pore formation kinetics in response to intrinsic or extrinsic apoptotic stimuli (staurosporine, tumor necrosis factorrelated apoptosis-inducing ligand (TRAIL)). We also detected that the onset of mitochondrial permeabilization frequently proceeded as a wave through the cytosol, and that the frequency of wave occurrence in response to TRAIL was reduced by inhibition of protein kinase CK2. Computational analysis by a partial differential equation model suggested that the spread of permeabilization signals could sufficiently be explained by diffusion-adsorption velocities of locally generated permeabilization inducers. Taken together, our study yielded the first comprehensive analysis of clonal cell-to-cell variability in apoptosis execution and allowed to visualize and explain the dynamics of MOMP in cells undergoing apoptosis.

Cell Death and Differentiation (2009) 16, 613-623; doi:10.1038/cdd.2008.187; published online 9 January 2009

Apoptosis is an evolutionary conserved cell death process that is fundamental to remove superfluous and damaged cells from the bodies of multicellular organisms. Impaired or excessive apoptosis has repeatedly been implicated as a major contributor to proliferative and degenerative diseases. Initiated by $\mathrm{BH}-3-$ only proteins of the $\mathrm{Bcl}-2$ protein family, mitochondrial outer membrane permeabilization (MOMP) and the subsequent activation of effector caspases are rapid key processes crucial for the efficient execution of apoptotic cell death.

Previous single-cell imaging studies using fluorescently tagged proteins showed that MOMP results in the coordinate release of mitochondrial intermembrane space proteins, including cytochrome-c (cyt-c) and Smac/Diablo, into the cytosol. The release was suggested to occur synchronously throughout the cytosol and with very fast kinetics, which seemed independent of the type of the apoptotic stimulus used, and also independent of downstream effector caspase activity. $^{1-5}$

Cytosolic cyt-c induces the rapid formation of the large multiprotein apoptosome complex, caspase- 9 activation and subsequent activation of effector caspases-3 and $-7^{6,7}$ In parallel, cytosolic Smac neutralizes $x$-linked inhibitor of apoptosis protein, the key inhibitor of caspases-9, -3 and -7 . $^{8}$ As long as all key components of the execution network are present, effector caspase activation proceeds as a rapid and kinetically largely invariant all-or-none process as shown by both fluorescence resonance energy transfer (FRET)-based single-cell imaging and computational systems analyses of HeLa cervical cancer and MCF-7 breast cancer cells. ${ }^{9,10}$ This irreversible switch-like behavior was recently attributed to system-inherent bistability emanating from feedback signaling loops during apoptosis execution. ${ }^{11}$ Fluorescent probes, such as cyt-c-GFP and GFP-based DEVDase substrates used in imaging studies, have been shown to behave very similar to endogenous proteins and to be reliable indicators for these processes. ${ }^{3,5,9,12}$

Of note, the lag time between addition of an apoptosisinducing drug and the apoptotic response frequently stretches from several hours to days. Furthermore, individual cells in a given cell population undergo apoptosis execution unpredictably at different, distinct times..$^{9,13,14}$ To avoid photobleaching and phototoxic side effects induced by prolonged imaging of the living specimen, single-cell studies of MOMP so far were limited to temporal resolutions of minutes. This lack of temporal resolution prevented to investigate whether mitochondrial permeabilization is subject to spatial coordination by processes such as localized production of signaling molecules and/or diffusion, which were previously described as important regulators of other cellular signaling pathways,

\footnotetext{
${ }^{1}$ Department of Physiology and Medical Physics, Royal College of Surgeons in Ireland, Dublin 2, Ireland; ${ }^{2}$ Systems Biology Group, Royal College of Surgeons in Ireland, Dublin 2, Ireland and ${ }^{3}$ Healthcare Division, Siemens Ireland, Leeson Close, Dublin 2, Ireland

*Corresponding authors: JHM Prehn, Department of Physiology and Medical Physics, Royal College of Surgeons in Ireland, 123 St. Stephen's Green, Dublin 2, Ireland. Tel: + 3531402 2255; Fax: + 3531402 2447; E-mail: prehn@ rcsi.ie and M Rehm, Department of Physiology and Medical Physics, Royal College of Surgeons in Ireland, RCSI York House, York Street, Dublin 2, Ireland. Tel: + 3531402 8563; Fax: + 3531402 2447; E-mail: mrehm@rcsi.ie

Keywords: apoptosis; cytochrome-c; caspases; mitochondrial outer membrane permeabilization; diffusion modeling

Abbreviations: cyt-c, cytochrome-c; FRET, fluorescence resonance energy transfer; MOMP, mitochondrial outer membrane permeabilization; PDE, partial differential equation; STS, staurosporine; TRAIL, tumor necrosis factor-related apoptosis-inducing ligand; CHX, cycloheximide; DRB, 5,6-dichloro-ribifuranosylbenzimidazole; PI, propidium iodide

Received 24.9.08; revised 12.11.08; accepted 27.11.08; Edited by D Vaux; published online 09.1.09
} 
such as the forwarding of phosphorylation signals and $\mathrm{Ca}^{2+}$ waves. $^{15-17}$

We here describe a novel approach combining rapid cellular imaging and computational modeling to remedy this limitation in the analysis of apoptotic signaling. Taking advantage of the synchrony of sibling cells in apoptosis onset, we established an imaging technique that is capable of predicting the time of MOMP for individual cells with high probability. This enabled us to monitor MOMP at seconds resolution in the absence of phototoxicity. In combination, we applied mathematical modeling to gain functional and mechanistic insight into the previously undescribed spatiotemporal coordination of mitochondrial permeabilization during apoptosis.

\section{Results}

Sibling cells undergo apoptosis synchronously. In previous studies, we have characterized MOMP and subsequent effector caspase-mediated apoptosis execution in real time by using fluorescence-based cell imaging. ${ }^{1,2,9}$ Activation of effector caspases and their resulting activity can be measured using a FRET approach using recombinant GFP-derived fusion proteins (Figure $1 \mathrm{a}$ and $\mathrm{b}$ ). As cells in a population do not commit apoptosis synchronously, ${ }^{13,14}$ we developed a live cell imaging approach that took advantage of the common mitotic history of dividing HeLa cells as a means of cell cycle-phase synchronization. On stage, we grew and monitored HeLa cells for times exceeding the cell cycle duration ( $26 \mathrm{~h}$ at experimental conditions) to observe cell division and the generation of daughter cells. This allowed us to follow sibling cell pairs and to record the exact time since division for each cell in the field of view. Even though the activation of apoptosis (MOMP or effector caspase activation) in a population of cells can stretch over many hours following stimulus addition, we observed that sibling cells underwent apoptosis execution within a surprisingly narrow time window, as observed by FRET analysis (Figure 1c). Random cell pairs instead were significantly less synchronous in undergoing apoptosis execution (Figure 1d).

Next, we quantified these responses in a large number of cells following exposure to strong apoptotic stimuli evoking similar cell death kinetics (Figure 1e and f). We used $1 \mu \mathrm{M}$ kinase inhibitor staurosporine (STS) to induce the intrinsic apoptotic pathway. STS was shown to induce the transcription/translation-dependent production of $\mathrm{BH} 3-o n l y$ proteins Puma and Noxa and to inhibit the post-translational phosphorylation of Bad. ${ }^{18,19}$ In our experimental setting, we could largely rule out the contribution of translation-dependent signaling in response to STS, as addition of translation inhibitor cycloheximide ( $\mathrm{CHX}$ ) did not impair the apoptotic response (Figure 1e). Extrinsic death receptor-dependent apoptosis was induced using $100 \mathrm{ng} / \mathrm{ml}$ tumor necrosis factorrelated apoptosis-inducing ligand (TRAIL). $\mathrm{CHX}$ was added in parallel to all TRAIL treatments to sensitize cells by suppressing parallel translation-dependent survival signaling. ${ }^{20}$ TRAIL-induced death receptor-mediated apoptosis leads to the activation of caspase- 8 and the cleavage and activation of BH3-only protein Bid. ${ }^{21}$
Our quantifications identified that sibling pairs in general were significantly more synchronous in effector caspase activation ( $\sim 20 \mathrm{~min}$ ) than random cell pairs, independent of activating the intrinsic or extrinsic pathway (Figure 1f). Similar results were obtained using these drugs at concentrations resulting in submaximal apoptotic responses (Figure $1 \mathrm{~g}$ ). We used HeLa cells for these analyses as their gap junction deficiencies restrict direct cell-to-cell communication by ion coupling or small second messengers. ${ }^{22}$ In a control experiment, neither cell-to-cell distance nor cell membrane contacts influenced the synchrony in apoptosis execution of sibling cells (not shown).

Furthermore, we analyzed whether the time elapsed since division influences the synchrony in apoptosis execution. First, we determined the cell-to-cell asynchrony in cell cycle progression. Following division of the mother cell, untreated sibling cells underwent their next cell division with a median asynchrony of $\sim 80 \min (n=163$ pairs analyzed). In response to treatment with either STS or TRAIL, we found that the asynchrony in apoptosis execution in sibling cells increased with progression through the cell cycle from an average of 10-20 min to 50-60 min (Figure 1h), supporting the hypothesis that the time elapsed since mitosis determines cell death synchrony. In addition, we analyzed whether this also influenced the responsiveness to STS- or TRAIL-induced apoptosis. We found that younger cells responded faster with apoptosis execution than older cells in response to both stimuli (Figure 1i). Grouping cells into $\mathrm{G} 1$ and $S$ phase on the basis of the previously published duration of cell cycle phases in HeLa cells ${ }^{23}$ yielded similar results, with cells in the G1 phase responding between 29 and 70 min earlier to TRAIL or STS than cells in S phase.

Kinetics of pore formation during mitochondrial outer membrane permeabilization. At present, it is unknown how fast the permeabilization of the outer mitochondrial membrane proceeds in living cells. Previously reported MOMP kinetics were measured at a temporal resolution of minutes and could not resolve the kinetics of the pore formation process: typical traces for the intracellular redistribution of fluorescently labeled cyt-c displayed as a fast process that already at the time of onset proceeded at maximal speed and were therefore considered to be independent of stimulus and dose $e^{2-5}$

On the basis of the high probability of sibling cells to commit apoptotic cell death in a very narrow time window, we were able to perform live cell imaging of mitochondrial permeabilization in response to STS or TRAIL using cyt-c-GFP at seconds resolution: Upon cell death of one sibling cell, the confocal scan field was adjusted to the sister cell and the respective fluorescence channel was sampled for short times at high frequencies (see Materials and Methods for details). No significant photobleaching could be observed following fast sampling of control cells for 20-30 min (Supplementary Figure 1).

When comparing cyt-c-GFP release in response to high doses of STS or TRAIL at fast sampling rates, we found that the time required for completion of STS-induced cyt-c-GFP release was longer than in response to TRAIL (Figure 2a and b). As in previous imaging studies, we quantitatively described 
a
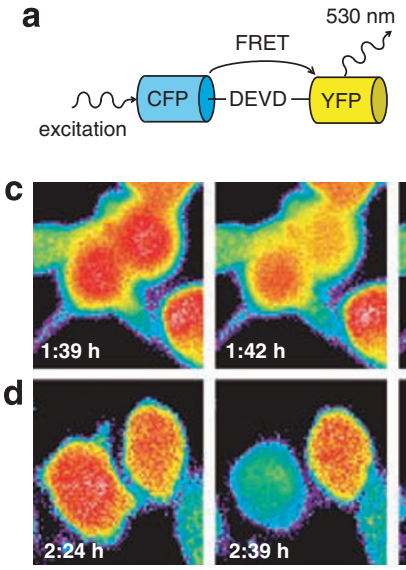

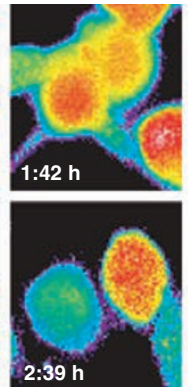

b
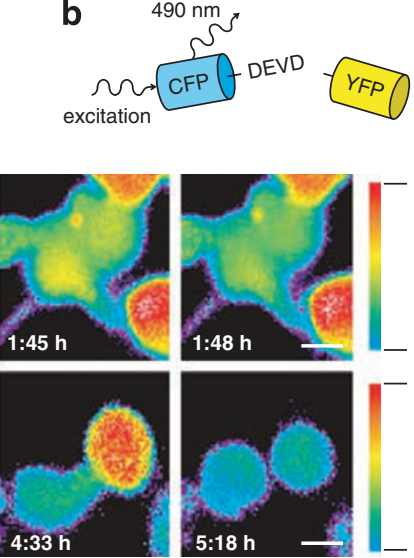

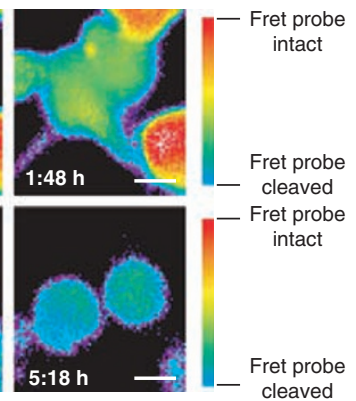

e

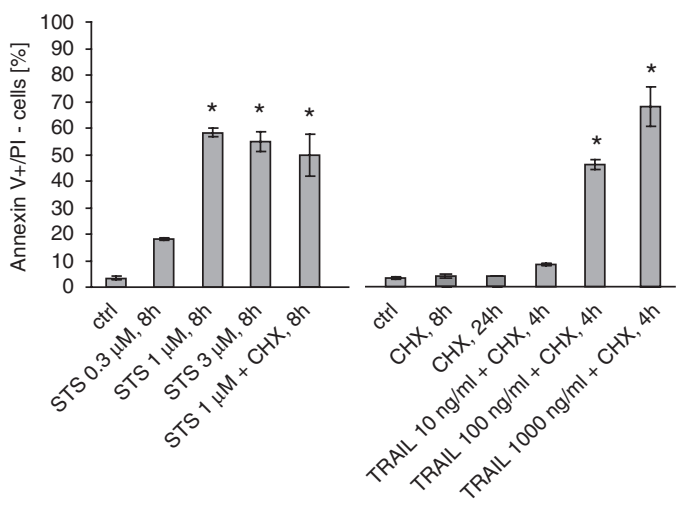

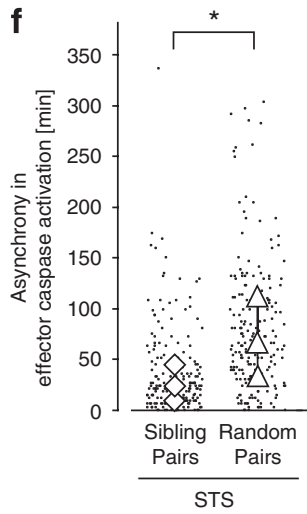

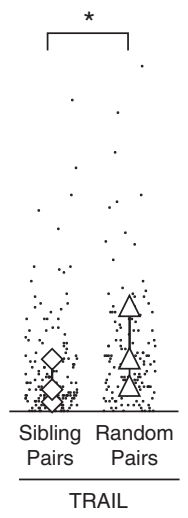

g

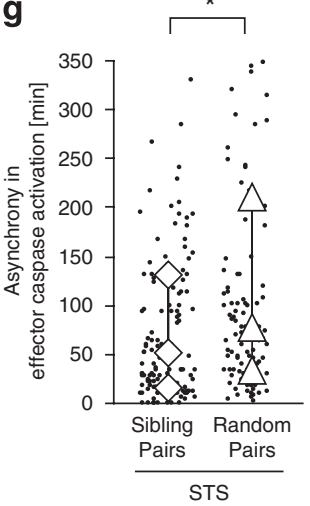

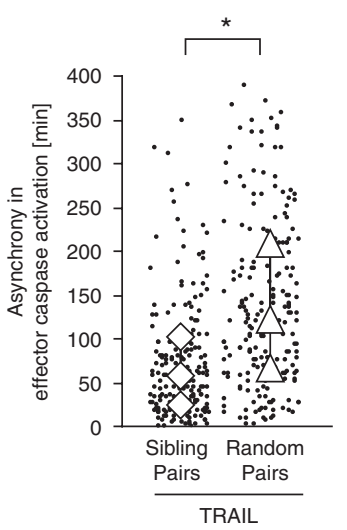

h

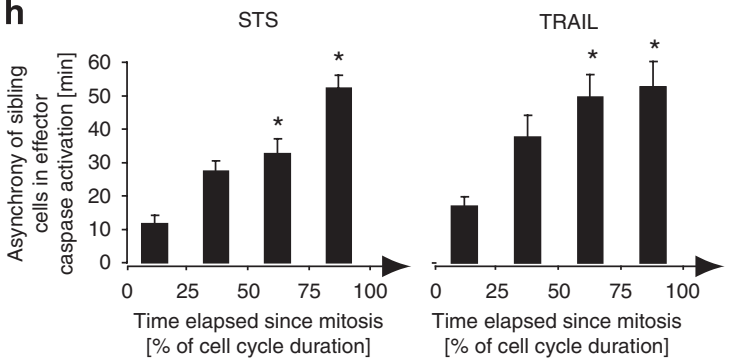

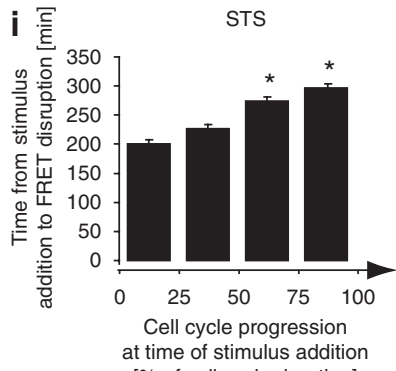

[\% of cell cycle duration]

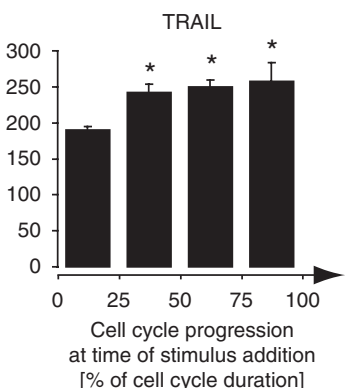

Figure 1 Synchronous apoptosis of sibling cells. (a, b) Measuring effector caspase activation using a recombinant DEVD-FRET probe. (a) The FRET probe is composed of cyan fluorescent protein (CFP), a linker containing a caspase-3/-7 specific cleavage site (DEVD), and yellow fluorescent protein (YFP). Upon CFP excitation, energy is transferred from the excited CFP to the acceptor YFP by FRET. (b) Cleavage of the linker region by effector caspase-3 results in FRET disruption and increased CFP emission. (c) FRET/YFP emission ratio images of a HeLa sibling cell pair treated with $3 \mu \mathrm{M}$ STS. Sibling cells undergo FRET probe cleavage synchronously. Time stamps indicate time after stimulus addition. Scale bar $=10 \mu \mathrm{m}$. (d) FRET/YFP emission ratio images of a random HeLa cell pair treated with $3 \mu \mathrm{M}$ STS. Cells undergo effector caspase activation asynchronously. Time stamps indicate time after stimulus addition. Scale bar $=10 \mu \mathrm{m}$. Please note that low FRET/YFP ratio intensities in cell peripheries in (c, d) are due to division of thin cell areas with low fluorescence intensities. ${ }^{9}$ (e) HeLa cells were treated with indicated concentrations of STS or TRAIL, respectively. Where indicated, CHX was added at $1 \mu \mathrm{g} / \mathrm{ml}$. Apoptosis was assessed by flow cytometric evaluation of Annexin-V FITC binding to phosphatidylserine in nonpermeabilized cells. Propidium iodide (PI) was used as a counterstain for necrosis/late apoptosis. Data are means \pm S.D. from $n=3$ parallel treatments each. Experiments were repeated at least once with similar results. $P<0.05$ : difference from untreated cells, ANOVA, and subsequent Tukey's test. (f) Quantification of cellular synchrony in apoptosis execution upon treatment with $3 \mu \mathrm{M}$ STS or $100 \mathrm{ng} / \mathrm{ml}$ TRAIL plus $1 \mu \mathrm{g} / \mathrm{ml} \mathrm{CHX}$ using live-cell FRET imaging. The individual data points represent temporal delays in effector caspase activation for sibling cell pairs or random cell pairs. Sibling cell pairs were significantly more synchronous than randomly selected cell pairs. Diamonds and triangles indicate median and first and third quartiles. A total of 193 and 167 cell pairs were analyzed per group for STS and TRAIL treatments, respectively. * Significant $\left(P=1.84 \times 10^{-16}\right.$ and $1.39 \times 10^{-8}$, respectively; MannWhitney U-test). (g) Synchrony of sibling cells at submaximal stimulus concentrations. Quantification of cellular synchrony in apoptosis execution upon treatment with $0.3 \mu \mathrm{M}$ STS or $10 \mathrm{ng} / \mathrm{ml}$ TRAIL plus $1 \mu \mathrm{g} / \mathrm{ml} \mathrm{CHX}$ using live-cell FRET imaging. Diamonds and triangles indicate median and first and third quartiles. A total of 138 and 184 cell pairs were analyzed per group for STS and TRAIL treatments, respectively. ${ }^{*}$ Significant $(P<0.05$; Mann-Whitney U-tests). (h) Asynchrony in apoptosis execution increases with cell cycle progression. According to the HeLa cell cycle duration at experimental conditions, cells were grouped into quarters of the cell cycle at the time of FRET probe cleavage. $n=27,46,35$, and 85 cell pairs analyzed per group for STS treatment. $n=23,41,56$, and 47 cell pairs analyzed per group for TRAIL treatment. Data represent average temporal asynchronies in effector caspase activation for sibling cell pairs or random cell pairs + S.E.M. *Significantly different to first group $(P<0.05$; ANOVA and subsequent Tukey's test). (i) Progression in cell cycle can delay apoptosis execution. Cells were grouped according to their progression through the cell cycle at the time of exposure to stimulus. $n=100,90,100$, and 96 cells analyzed per group for STS treatment $(1 \mu \mathrm{M}) . n=100,116,90$, and 28 cells analyzed per group for treatment with TRAIL and CHX $(100 \mathrm{ng} / \mathrm{ml}$ and $1 \mu \mathrm{g} / \mathrm{ml}$, respectively). Data are shown as means + S.E.M. *Significantly different to first group $(P<0.05$, ANOVA and subsequent Tukey's test) 

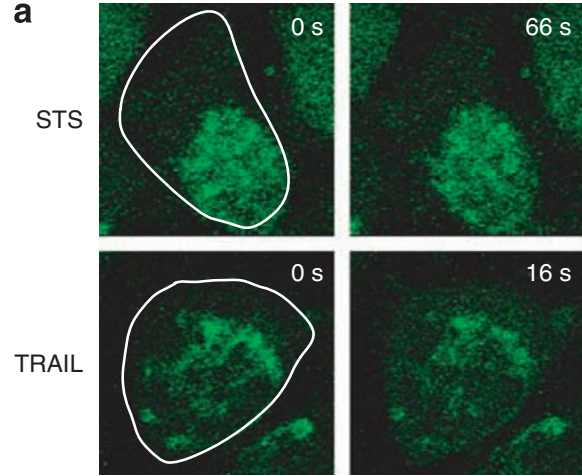
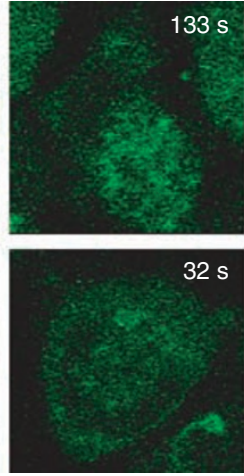
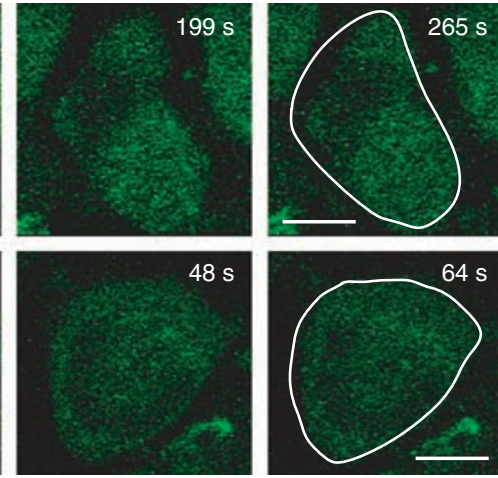

b

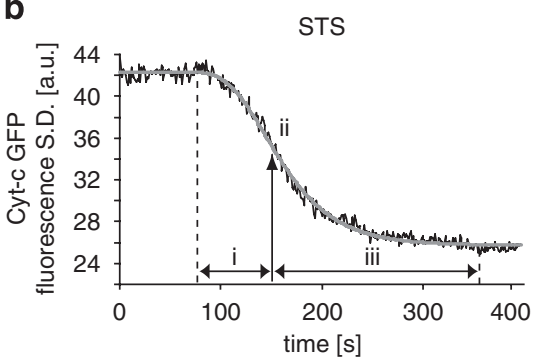

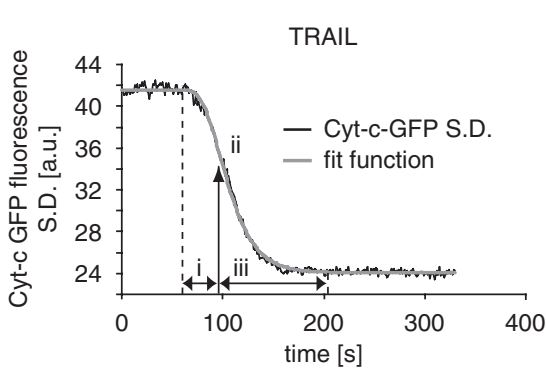

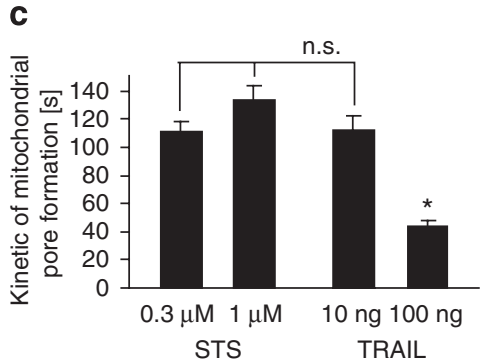

Figure 2 Analysis of pore formation kinetics in the outer mitochondrial membrane. (a) Differential kinetics of STS and TRAIL induced cyt-c-GFP release. Images show representative HeLa cells sampled at $1 \mathrm{~s}$ temporal resolution releasing cyt-c-GFP synchronously throughout the whole cell. Cells were treated with STS (1 $\mu \mathrm{M}$ ) or TRAIL and $\mathrm{CHX}(100 \mathrm{ng} / \mathrm{ml}$ and $1 \mu \mathrm{g} / \mathrm{ml}$, respectively). Time stamps are synchronized to the onset of cyt-c-GFP release. Scale bars $=8$ and $10 \mu \mathrm{m}$, respectively. (b) Mathematical analysis of mitochondrial pore formation kinetics. The cyt-c release kinetics for the cells shown in (a) were plotted as the change in cellular fluorescence redistribution (S.D.). Cyt-c release comprises a fast onset (i), passes the point of inflexion (ii), and a slower phase (iii) leading into the final baseline. Traces were fitted with a modified Gompertz function (gray). The points of inflexion specify the time when the speed of cyt-c-GFP release is maximal (black arrow). Onset of mitochondrial permeabilization (dashed line) was defined as the time when the fit function deviates by $2 \%$ from the initial baseline. The kinetic of mitochondrial pore formation was defined as the time from the onset to the point of inflexion. Time zero corresponds to the onset of cyt-c release. See Materials and Methods for further details. (c) The kinetics of mitochondrial pore formation were measured for $n=18,10,13$, and 30 HeLa cells following treatment with STS ( $1 \mu \mathrm{M} ; 0.3 \mu \mathrm{M})$ or TRAIL ( $100 \mathrm{ng} / \mathrm{ml} ; 10 \mathrm{ng} / \mathrm{ml})$ plus $1 \mu \mathrm{g} / \mathrm{ml}$ CHX, respectively. Data are shown as mean + S.E.M. *Significant, $P=0.003$; n.s., nonsignificant (ANOVA and subsequent Tukey's test)

the redistribution of cyt-c-GFP by plotting the change in the standard deviation of the cellular GFP fluorescence distribution over time (Figure $2 \mathrm{~b}$ ). ${ }^{2,4}$ At seconds resolution, we could resolve two distinct kinetic phases during cyt-c-GFP redistribution: an initiation phase during which the actual kinetics of mitochondrial pore formation could be determined and a second phase of kinetically unimpaired cyt-c redistribution (Figure 2b). Following an initial baseline, pore formation was rapidly initiated (i), passed the point of fastest cyt-c release (ii) into a slower phase (iii) until a final baseline was reached that resembled complete cyt-c-GFP redistribution. The release processes proceeded faster during TRAILinduced MOMP and resembled asymmetric sigmoid traces that correspond well to the mathematical behavior of a modified Gompertz function allowing for sigmoid asymmetry (Figure 2b):

$$
y=-a * \exp \left(-\exp \left(-k\left(t-t_{c}\right)\right)\right)+b
$$

where $t$ denotes time, $a$ the amplitude, $k$ the decay rate, and $b$ the $y$ offset. $t_{c}$ denotes the time of inflexion, which represents the mitochondrial permeabilization allowing for fastest cyt-c release.

Extracting the time from onset of cyt-c-GFP release until the point of inflexion from the fitted traces, we could determine the time required for mitochondrial pore formation to reach its highest efficiency for cyt-c-GFP release. Analysis of the data identified that pore formation during apoptosis induced by STS appears to be independent of using high or low doses. In contrast to previous assumptions, at high doses of TRAIL, pore formation kinetics in vivo were approximately threefold faster than during apoptosis induced by submaximal TRAIL doses or STS (Figure 2c).

Spatial waves of MOMP induction detected by cyt-c-GFP release. At rapid sampling rates, we frequently observed that MOMP-induced cyt-c-GFP release started in subcellular mitochondrial regions and from there continued to spread throughout the cell body (Figure 3a, Supplementary Video 1). At high TRAIL doses, MOMP waves were much more prominent when compared with low-dose treatments $(63 \%$ versus $18 \%$ of analyzed cells, i.e., $n=38$ and 39 cells analyzed per group, respectively) (Figure $3 b$ ). In contrast, treatment with kinase inhibitor STS at high doses strongly diminished the occurrence of MOMP waves (26\% versus $71 \%$ of analyzed cells, i.e., $n=31$ and 29 cells analyzed per group, respectively) (Figure $3 b$ ).

We therefore further investigated whether a molecular mechanism could be identified explaining the occurrence of MOMP waves. It was shown previously that TRAIL may induce the formation of death receptor aggregates and the 

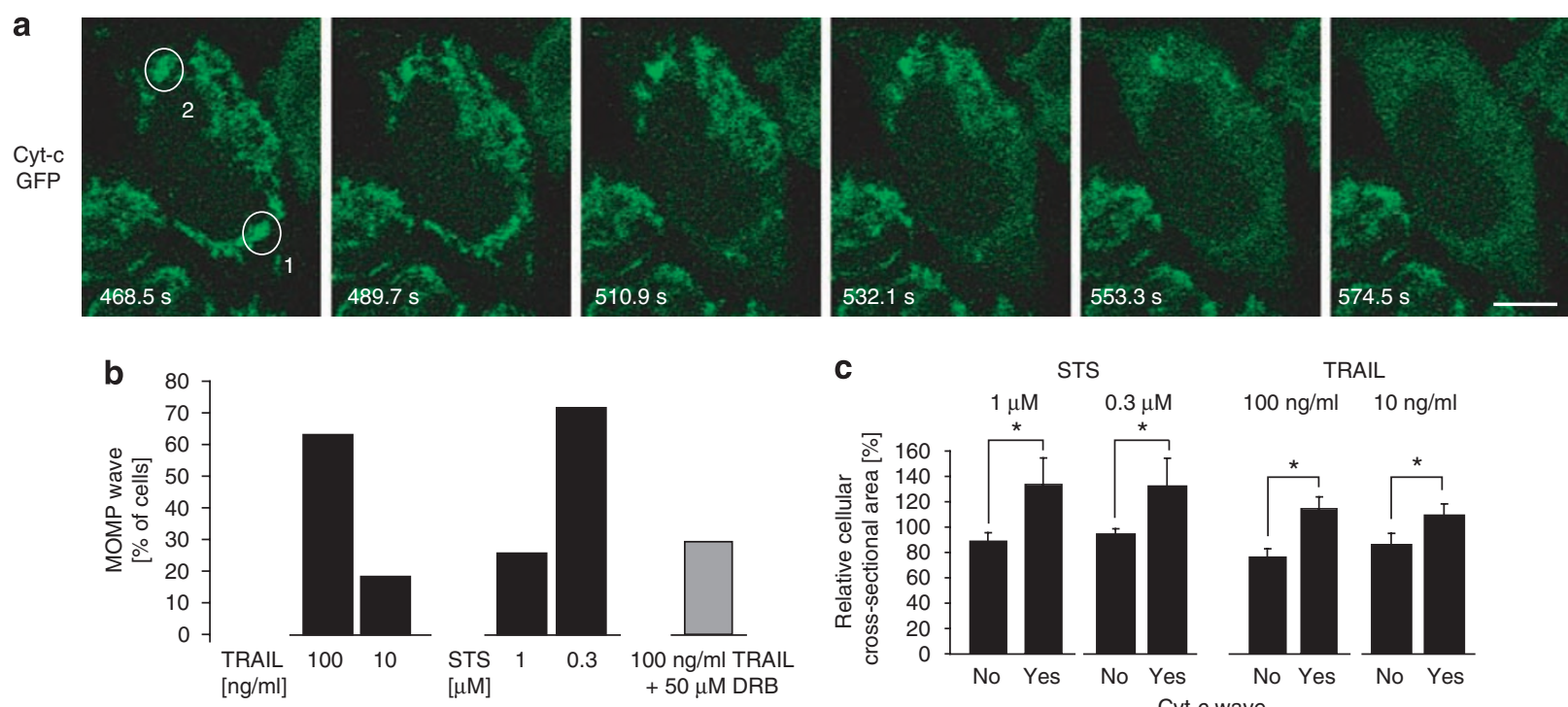

STS

TRAIL

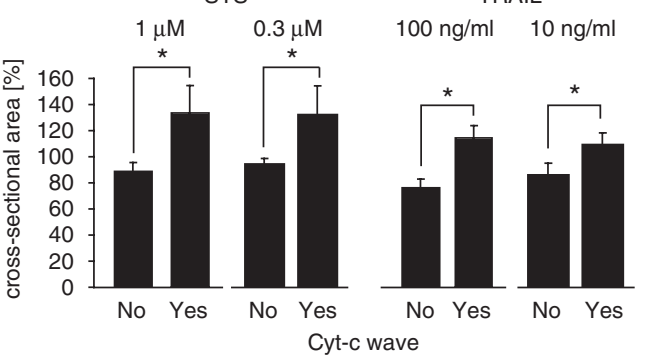

e
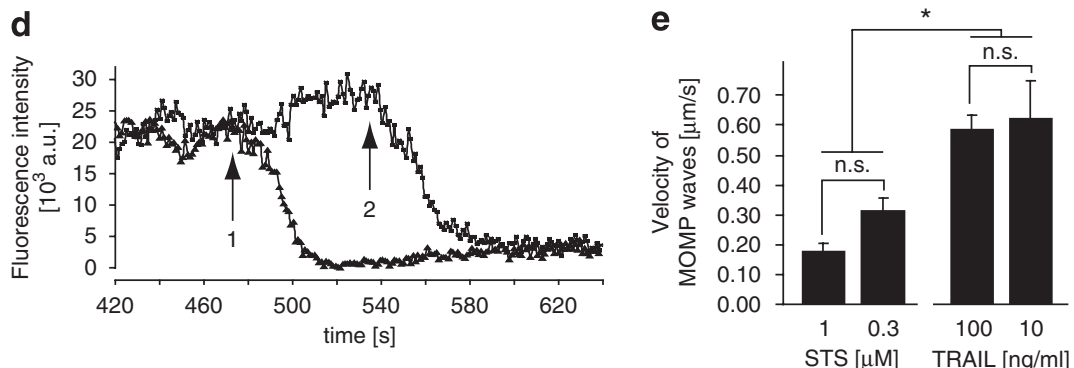

Figure 3 Detection and analysis of spatial waves of MOMP induction. (a) Intracellular waves of MOMP induction detected by the release onset of cyt-c-GFP. Images show a representative HeLa cell releasing cyt-c-GFP from the mitochondria into the cytosol. From the region of onset (region 1), cyt-c-GFP release proceeded as a wave through the cell. Region 2 shows the last region to release cyt-c-GFP. The cell shown was exposed to $100 \mathrm{ng} / \mathrm{ml} \mathrm{TRAlL}$ and $1 \mu \mathrm{g} / \mathrm{ml} \mathrm{CHX} 56 \mathrm{~min}$ before the onset of fast sampling at $1.06 \mathrm{~s}$ resolution. Time stamps show time after onset of fast sampling. Scale bar $=10 \mu \mathrm{m}$. A related video file is available online as Supplementary Video 1. (b) Probability of MOMP waves. All cells measured were analyzed for the occurrence of waves of MOMP initiation in response to STS $(1 \mu \mathrm{M}$ or $0.3 \mu \mathrm{M})$, TRAIL/CHX (100 ng/ml or $10 \mathrm{ng} / \mathrm{ml}$ TRAlL plus $1 \mu \mathrm{g} / \mathrm{ml} \mathrm{CHX}$, respectively), or TRAIL/CHX subsequent to pretreatment with $50 \mu \mathrm{M}$ DRB, respectively. $n=31$ and 28 or 38,39 , and 27 single cell responses from $n=3,4,2$, and 3 independent experiments were analyzed per group, respectively. (c) Waves of MOMP induction are more frequent in large cells. Cells were grouped according to the occurrence of MOMP waves and analyzed for the area covered in confocal scans. The average size of all cells measured served as the reference point of $100 \%$. Data are shown as means + S.E.M. from $n=23$ and 8 cells for $1 \mu \mathrm{M}$ STS treatment, $n=10$ and 18 cells for $0.3 \mu \mathrm{M} \mathrm{STS} \mathrm{treatment,} n=14$ and 24 cells for treatment with $100 \mathrm{ng} / \mathrm{ml}$ TRAIL and $1 \mu \mathrm{g} / \mathrm{ml} \mathrm{CHX}$, or $n=32$ and 7 cells for treatment with $10 \mathrm{ng} / \mathrm{ml} \mathrm{TRAIL} \mathrm{and} 1 \mu \mathrm{g} / \mathrm{ml} \mathrm{CHX}$, respectively. ${ }^{*}$ Significant $(P<0.05$; Student's $t$-test). (d) Kinetic analysis of MOMP waves by measuring the onset of cyt-c-GFP release. For the cell shown in (a), mitochondrial GFP fluorescence was segmented from the cytosolic area by thresholding. The region of onset of cyt-c-GFP release (region 1) and the last region to show release (region 2) were analyzed for their loss in mitochondrial cyt-c-GFP fluorescence. Time scale shows time after onset of fast sampling. The numbered arrows indicate the onset of cyt-c-GFP release in the respective region. (e) Quantification of MOMP wave velocity. The velocity was calculated by dividing the distance the MOMP wave traveled by the delay in release onset of cyt-c-GFP in the respective cellular regions. Data are shown as

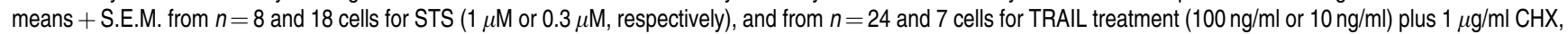
respectively. *Significant $(P<0.05 ; \mathrm{NS}=$ nonsignificant (ANOVA and subsequent Tukey's $t$-test)

recruitment of apoptotic adapter molecules into spatially confined regions, such as lipid rafts and 'caps'. ${ }^{24}$ However, in our experimental system, we could not observe any such pronounced receptor accumulation at the cell surfaces of HeLa cells during apoptosis induced with $100 \mathrm{ng} / \mathrm{ml}$ TRAIL when performing immunofluorescence stains against death receptors 4 or 5 (not shown). As kinase cascades can be initiated following TRAIL treatment ${ }^{20}$ and as high doses of kinase inhibitor STS reduced the overall amount of cells showing MOMP waves during intrinsic apoptosis, we hypothesized that kinase signaling might play a more general role in contributing to spatial anisotropies of MOMP induction. Correspondingly, for death receptor-induced apoptosis, it was suggested previously that dephosphorylation of the caspase-
8 BH3-only target Bid might enhance its susceptibility to caspase-8-dependent cleavage. ${ }^{25,26}$ We therefore pretreated HeLa cells with $50 \mu \mathrm{M}$ 5,6-dichloro-ribifuranosylbenzimidazole (DRB), a specific inhibitor of the Bid-phosphorylating serine/threonine kinase $\mathrm{CK} 2 .^{26-28}$ DRB resulted in a significant decrease of Bid phosphorylation without affecting the total amount of Bid as observed by western blotting (data not shown). We used this inhibitor instead of RNAi approaches to eliminate CK2 activity, as CK2 exists in multiple isoforms and comprises two separate catalytic subunits. ${ }^{29}$ When analyzing these cells for MOMP waves following exposure to high TRAIL doses, we indeed found that waves were only rarely observed (30\% of 27 cells analyzed) (Figure $3 b$ ). DRB alone did not induce any significant cell 
death during the duration of the experiments. These results indicate not only that MOMP can be subject to a spatially inhomogeneous induction, but also that the spatiotemporal coordination of MOMP induction can dose dependently depend on whether apoptosis is initiated intrinsically or extrinsically. Furthermore, the spatial coordination of MOMP induction might strongly depend on kinase activities.

We next asked whether the occurrence of these waves was also influenced by cellular size. Cellular size was determined as the relative cellular cross-sectional area from the number of pixels individual cells covered in the confocal scan areas. For STS and TRAIL treatment at both high and low doses, we found that cells showing waves of MOMP induction were significantly larger than cells in which cyt-c-GFP was released homogeneously throughout the cell (Figure 3c).

As a directly accessible measure for the speed of the MOMP waves, we determined their end-to-end velocities from the image sequences. Mitochondrial regions were segmented from the cytosolic background, and the time required for the onset of cyt-c-GFP release to travel through the cell body from one region to the most distal region was calculated (Figure $3 a$ and $d$ ). For comparison, traces for a cell not displaying a MOMP wave are provided as Supplementary Figure 2. The end-to-end velocities of MOMP waves were determined by dividing the distance between the first and last region showing release by the time required to cross this distance. Even though differences in the subcellular distribution of cellular organelles and cellular geometry can contribute to cell-to-cell differences, we found that the apparent velocities of MOMP waves in response to extrinsic TRAILinduced apoptosis $(0.58 \pm 0.06 \mu \mathrm{m} / \mathrm{s}$ to $0.62 \pm 0.13 \mu \mathrm{m} / \mathrm{s})$ were significantly higher than during STS-induced intrinsic apoptosis $(0.17 \pm 0.03 \mu \mathrm{m} / \mathrm{s}$ to $0.31 \pm 0.05 \mu \mathrm{m} / \mathrm{s})$ (Figure $3 e$ ).

Taken together, these results show that MOMP can be spatiotemporally coordinated, and that this coordination is differentially regulated during intrinsic STS-induced or extrinsic TRAIL-induced apoptosis and might strongly depend on the phosphorylation status of BH3-only proteins and/or other upstream regulators in these pathways.

Mathematical diffusion modeling of MOMP waves. For the scenario of TRAIL-induced apoptosis, we used mathematical diffusion modeling to investigate whether the observed waves of MOMP induction could be explained mechanistically. The modeled situation differs in various aspects from classical simple diffusion scenarios: (i) a closed system for the diffusion process, representing an intact HeLa cell (approximately $30 \mu \mathrm{m}$ as determined by confocal microscopy), was assumed; (ii) on the basis of our experimental evidence, we assumed a localized generation of MOMP-inducing factors, which during TRAIL-induced apoptosis could represent a local source of truncated BH3only protein Bid (tBid); (iii) a production kinetic of the MOMP inducer at this location was mathematically implemented in the model. As reported in a previous study ${ }^{30}$ and measured in additional control experiments in HeLa cells (data not shown), the complete conversion of Bid into the MOMP inducer tBid in individual living cells is a rapid process and can be achieved in approximately $20 \mathrm{~min}$. The spatiotemporal distribution of the MOMP inducer was modeled on the basis of diffusion physics by using a partial differential equation (PDE) derived from Fick's second law for nonsteady-state diffusion. ${ }^{31} \mathrm{~A}$ comprehensive description of the mathematics underlying the model generation and the respective MATLAB code can be found as Supplementary Information 3 and 4.

First, we examined the propagation velocities of MOMPinducing thresholds. We assumed that a local tBid concentration of $5-20 \%$ of the total Bid is sufficient for inducing mitochondrial permeabilization, as very low MOMP thresholds for tBid were described in vivo and in vitro previously. ${ }^{30,32}$ The model suggested that in this range, the end-to-end velocities are largely independent of the required threshold of the MOMP inducer (Figure 4a). However, the diffusion model was not able to reproduce the experimental data: The calculated end-to-end velocities of MOMP waves exceeded those measured during TRAIL-induced apoptosis (Figure 4a), suggesting that an additional process needed to be considered.

We therefore took into account that the MOMP inducer, while diffusing through the cell, contributes to mitochondrial permeabilization by adsorption to mitochondria and biochemically interacting with pro- and antiapoptotic Bcl-2 family members. Upon adsorption to mitochondria, the MOMP inducer is removed from the diffusing cytosolic pool and furthermore was assumed to be immobilized. The mathematical implementation of this process is explained in the expanded model description (Supplementary Information 3).

This extended model indeed was sufficient to reproduce the experimentally observed end-to-end velocities of MOMP waves during TRAIL-induced apoptosis (Figure 4a). Plotting the signal progression through time and space, our calculations also suggested that the MOMP waves may accelerate while progressing through the cells, as the slopes of the propagation time versus distance were not constant but rather decreased (Figure 4b). Mathematically, this behavior emanated from the conditions of a localized production and subsequent diffusion within a closed system. ${ }^{33}$

We therefore analyzed whether this model hypothesis was supported by the experimental data. Following up on the determination of end-to-end velocities of MOMP waves (Figure 3), we next analyzed the subcellular progression of the wave fronts in kymographs (Figure 4c). Such kymographs closely corresponded to the calculated profiles (Figure 4b) and allowed to determine wave velocities in $3 \mu \mathrm{m}$ intervals along the wave front. Wave velocities in response to $100 \mathrm{ng} / \mathrm{ml}$ TRAIL were then plotted against distance traveled (Figure 4d). This analysis supported the model prediction that MOMP waves accelerate with the distance traveled. It also agreed well with velocities calculated from profiles of the modeled wave fronts (Figure $4 b$ and $d$ ). Similar results were also obtained at submaximal TRAIL stimulation (10 ng/ $\mathrm{ml})$ as well as maximal and submaximal doses of STS $(1 \mu \mathrm{M}$ and $0.3 \mu \mathrm{M}$, respectively) (data not shown). Only at large distances from the point of onset the calculated velocities started to deviate from the experimental data (Figure 4d). This was expected, as our mathematical calculation suggests that wave velocities excel close to the far-end boundary of the cell. Furthermore, experimental measurements are subject to additional variability, such as variability in cell length and shape, as well as cell-to-cell differences in mitochondrial 
distribution patterns, which cannot be accommodated by a simplistic model. Still, the modeling approach combined with the experimental analyses supported the assumption that MOMP waves could depend on the localized production and a diffusion-adsorption process of MOMP inducers, such as tBid and other BH3-only proteins, within a closed system.

Propagation velocities of MOMP waves may depend on the production kinetics of MOMP inducers. Interestingly, the velocities of MOMP waves were calculated to be largely
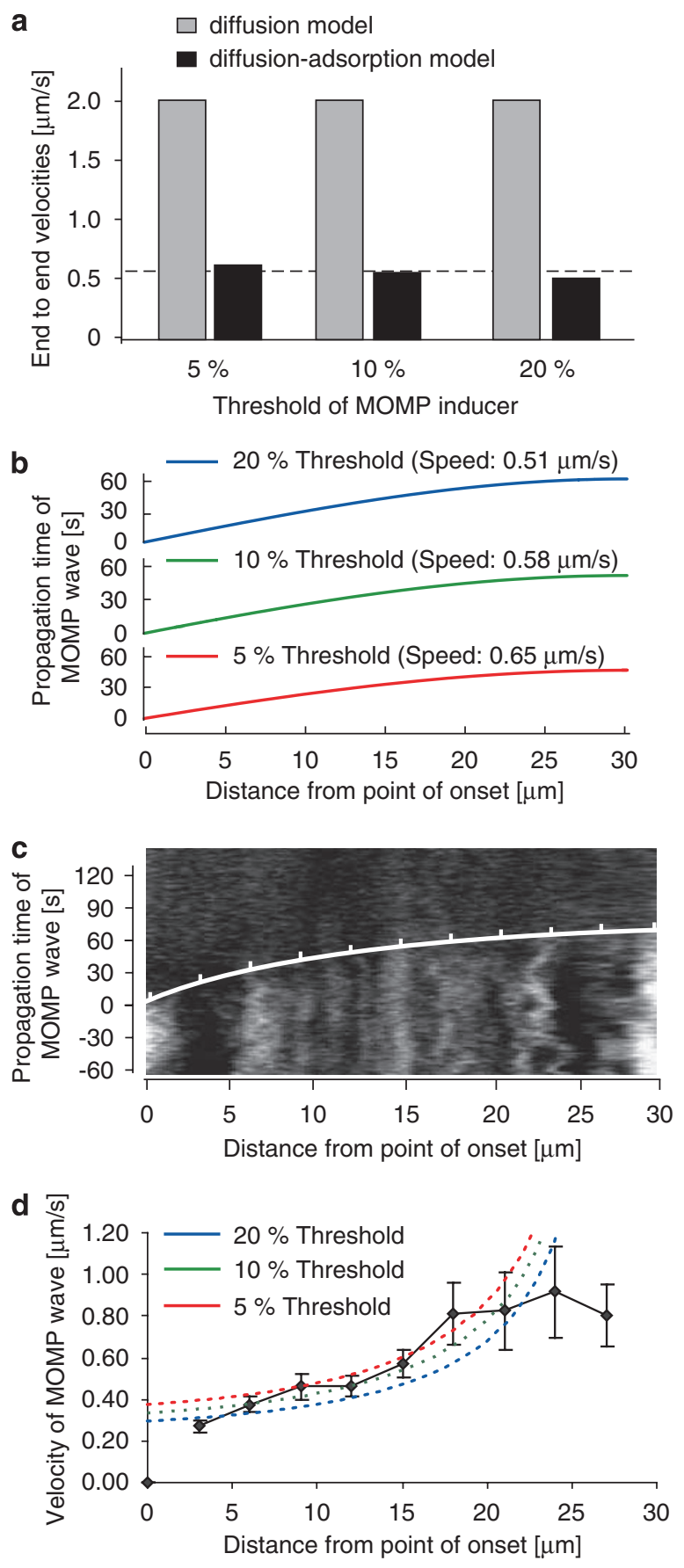

independent of the threshold required for mitochondrial permeabilization, as $\mathrm{tBid}_{\text {MOMP }}$ spatiotemporally distributed largely identical for thresholds up to approximately $70 \%$ (Figure 5a). Furthermore, as the adsorption of tBid to mitochondria is a rapid process, the model suggests that

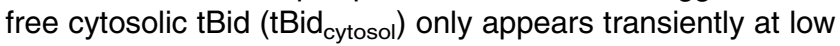
concentrations (Figure $5 \mathrm{~b}$ ). We finally analyzed how individual production rates of MOMP inducers (see point (iii) of the model generation above and Equation 5 in Supplementary Information 3) influence the propagation velocities of MOMP waves. Corresponding to the results shown in Figure $4 \mathrm{a}$, only the diffusion-adsorption model was able to reproduce propagation velocities in the experimentally measured range (Figure $5 \mathrm{c}$ ). The diffusionadsorption model also suggests that velocities below $0.2 \mu \mathrm{m} /$ $\mathrm{s}$ (as observed during STS-induced apoptosis) may be indicative of production times of MOMP inducers in the range of hours in this scenario (Figure $5 \mathrm{c}$ ).

\section{Discussion}

On the basis of the synchronous responses of cells with a common mitotic history, we developed an approach that allows for the fast sampling of living, intact cells, which enabled new insights into the spatial and temporal coordination of mitochondrial permeabilization during apoptosis. The synchrony of sibling cells in undergoing effector caspase activation was found to be most prominent early after mitosis and to decrease significantly with cell cycle progression. Nevertheless, even far into the cell cycle, their responses were surprisingly well correlated when considering the complexity of the signaling events required before responding with MOMP and apoptosis execution. It is reasonable to assume that, after a cell division, similar protein identities and

Figure 4 Mathematical diffusion analysis of spatial MOMP induction. (a) A diffusion-adsorption model can reproduce the propagation velocities of MOMP waves. End-to-end velocities of MOMP waves were calculated using a diffusion model (gray) or a diffusion-adsorption model (black). Velocities are shown for threshold concentrations of 5,10 or $20 \%$ MOMP inducer. The dashed line indicates the experimentally observed end-to-end velocities in response to $100 \mathrm{ng} / \mathrm{ml}$ TRAIL. Different thresholds propagate with largely identical velocities. (b) Spatiotemporal propagation of tBid-induced MOMP waves using the diffusion-adsorption model. The times required until thresholds of 5,10 , and $20 \%$ of MOMP-contributing tBid (tBid ${ }_{\text {MOMP }}$ ) are reached at different intracellular locations are shown. tBid production is located at position $0 \mu \mathrm{m}$. The indicated propagation speeds were calculated end

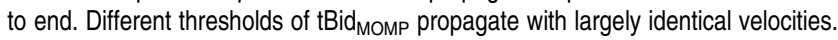
(c) Kymograph analysis of MOMP wave fronts. The exemplary kymograph shows progression of cyt-c-GFP release against distance for a representative HeLa cell treated with $100 \mathrm{ng} / \mathrm{ml}$ TRAIL plus $1 \mu \mathrm{g} / \mathrm{ml} \mathrm{CHX} \mathrm{(see} \mathrm{Figure} \mathrm{3a).} \mathrm{High} \mathrm{intensities}$ represent mitochondria still containing cyt-c-GFP. Over time, mitochondria along the kymograph line release cyt-c-GFP into the cytosol, resulting in a loss of signal. The white line outlines the wave front and can be compared with calculated MOMP wave profiles in (a). White tick marks indicate $3 \mu \mathrm{m}$ distances. (d) MOMP waves accelerate with the distance traveled. Change of velocity with distance for all MOMP waves measured in HeLa cells treated with $100 \mathrm{ng} / \mathrm{ml}$ TRAIL plus $1 \mu \mathrm{g} / \mathrm{ml} \mathrm{CHX}$ were analyzed. Velocities were calculated for $3 \mu \mathrm{m}$ intervals along the wave fronts in the generated kymographs. Average velocities were calculated from $n=10-23$ measurements for the individual data points and shown + S.E.M. Experimentally determined velocities can be compared with model predictions on wave velocities at 5,10 , or $20 \%$ thresholds for tBid MOMP (dotted lines). Modeled wave velocities were obtained from temporal derivatives of the calculated wave-front profiles shown in (a) 
protein concentrations may be retained in sibling cells. Later, cell-to-cell variability may arise from noise and differences in transcription, translation, modification and degradation

a
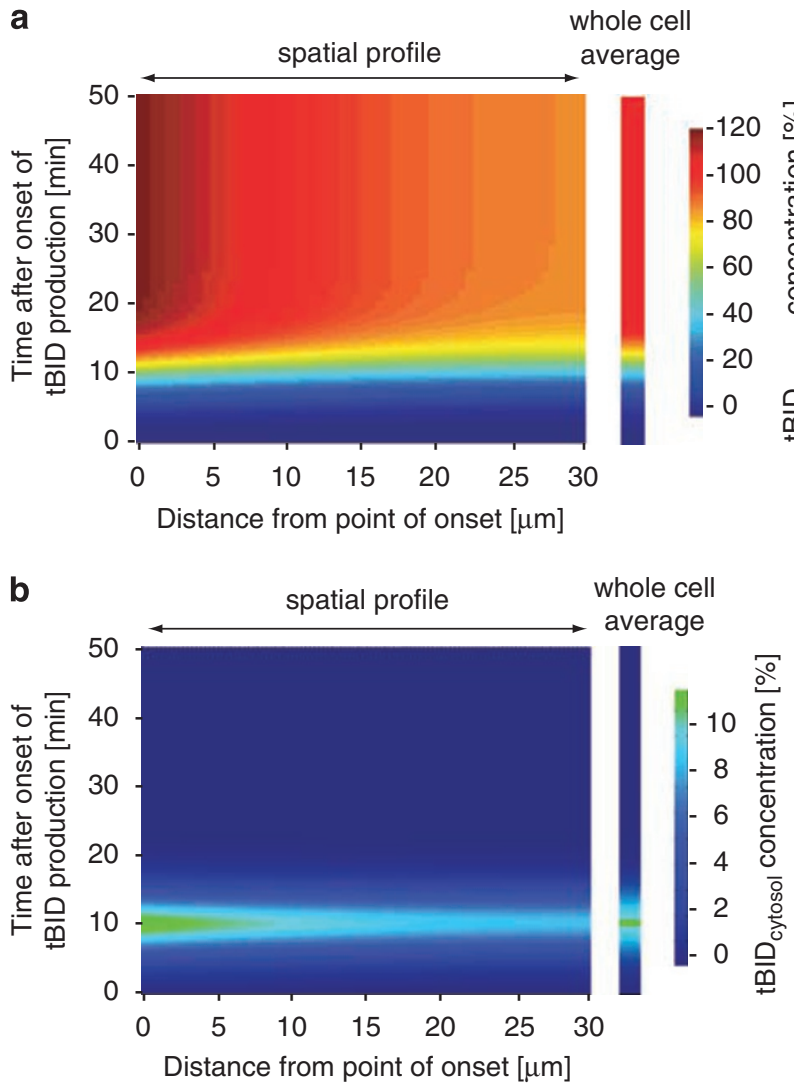

C

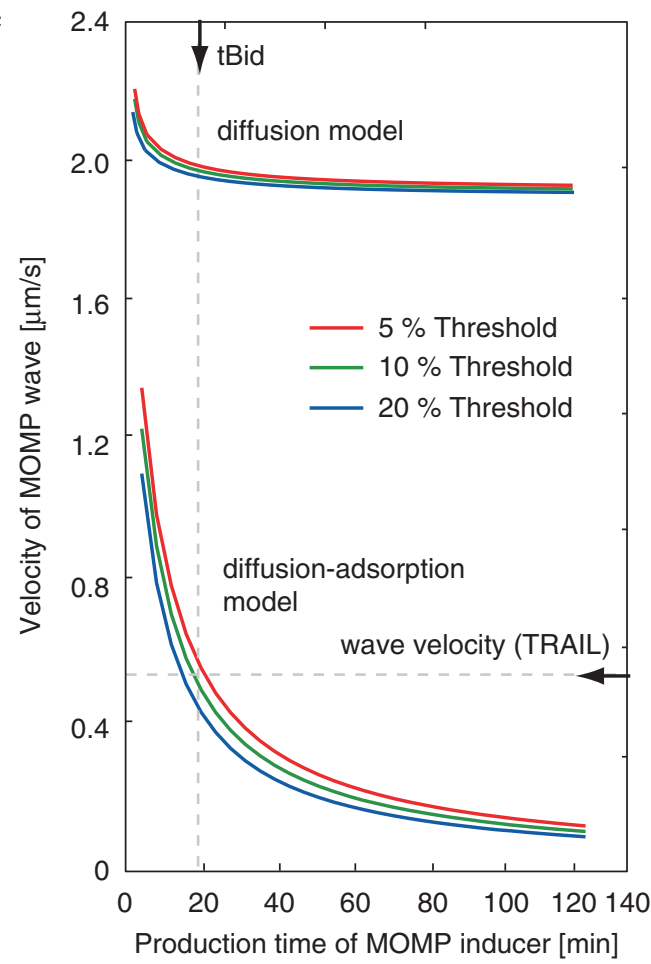

processes as well as from cell migration and cell-to-cell communication events. In this context, it was also shown recently that cellular protein profiles can be inherited beyond cell cycle durations. ${ }^{34}$ From a technical point of view, our approach avoids the requirement of chemical cell synchronization, ${ }^{14,35}$ which may induce additional stress responses in cells, including the activation of apoptosis by the chemical compounds themselves.

We used GFP-fused cyt-c to describe the kinetics of mitochondrial permeabilization during apoptosis at high temporal resolution. Cyt-c-GFP is a well-established and reliable probe to detect MOMP, and the GFP fusion was suggested not to impair the kinetic behavior of native cyt-c. ${ }^{4,5}$ Furthermore, the molecular weight of cyt-c-GFP closely resembles other mitochondrial intermembrane space proteins released into the cytosol during apoptosis, such as dimeric Smac.

Rapid sampling of cyt-c-GFP release in intact living cells could for the first time describe stimulus-dependent kinetics of spatial waves of MOMP induction. Similarly, although described for large permeabilized cells, waves of $\mathrm{Ca}^{2+}$ signals were implicated in cell death signaling processes that induce mitochondrial permeability transition, ${ }^{17}$ suggesting contribution of spatial signaling also during other cell death processes/paradigms. In our study, the frequency of spatial waves of MOMP induction was strongly affected by kinase inhibitors that were reported to affect the phosphorylation status of BH3-only proteins involved in STS- and TRAILinduced apoptosis, ${ }^{18,25,26}$ highlighting that de-/phosphorylation processes could play a crucial role in the localized activation of the apoptosis program.

Several insightful systems modeling studies, either purely theoretical or in combination with experimental approaches, were already conducted to elucidate temporal dynamics of intracellular signaling, response thresholds and stability properties of apoptotic networks by using mathematical models on the basis of differential equations. ${ }^{10,11,36-39}$ Time-lapse fluorescence imaging of living cells, a noninvasive method appropriate to measure the real-time kinetics of apoptotic processes, can directly be linked with mathematical models of intracellular apoptosis signaling. ${ }^{10,40}$ The mathematical model proposed here for the mechanism of spatial MOMP waves builds on the lex parsimoniae, otherwise also

Figure 5 Spatiotemporal distribution and propagation velocities of MOMP thresholds. Spatiotemporal distribution profiles of the mitochondrially adsorbed (a) and remaining cytosolic (b) fractions of tBid shown in a pseudocolor scale. The concentration scale was normalized to $100 \%$ reference concentration reached if tBid was completely produced and evenly distributed in the whole cell. tBid production is located at position $0 \mu \mathrm{m}$. (a) Times when mitochondrially adsorbed tBid concentrations are reached at different positions are shown. Mitochondrially adsorbed tBid was considered immobilized. (b) The free cytosolic tBid fraction was calculated to be present only transiently at relatively low concentrations. Cytosolic tBid concentrations are diminished due to mitochondrial adsorption. (c) Modeled propagation velocities of MOMP waves depend on the production kinetic of the MOMP-inducing factor. Velocities for thresholds of 5,10 , and $20 \%$ are shown against different production times of the MOMP inducer. The dashed gray lines indicate the experimentally observed production kinetic for MOMP inducer tBid and the experimentally observed MOMP wave velocities. The model predicts lower velocities of MOMP waves upon production times of MOMP inducers in the range of hours 
known as the principle of Occam's razor. This heuristic maxim of scientific theory proposes to use as few assumptions and free parameters as possible to explain the observed processes. We therefore assumed that production kinetics and diffusion-adsorption processes of MOMP inducers were sufficient to explain the experimentally detected spatial spread cyt-c-GFP release. Our model correlated well with the experimental evidence and was sufficient to predict subcellular wave-front profiles. Mathematical modeling also suggested that the velocity of the MOMP wave is largely independent of the $\mathrm{BH}$-only threshold required for MOMP induction. We were able to show previously in vivo and others in vitro that minute amounts of tBid may be sufficient to induce MOMP, ${ }^{30,32}$ suggesting that the threshold required in vivo must be very low indeed. As long as cells do not express pathologically elevated levels of antiapoptotic Bcl-2 family proteins, this conversely suggests that the cellular decision as to activate MOMP or not is probably not so much regulated at the mitochondrial level but rather upstream, that is, at the level of the generation of MOMP-inducing factors.

Interestingly, we also observed that the pore formation process itself appeared to proceed faster in response to high TRAIL doses when compared with submaximal stimulation or high and low doses of STS. It is conceivable that dosedependent activation of caspase-8, which was recently described by us in HeLa cells, might contribute to this. ${ }^{41}$ The different MOMP kinetics in response to TRAIL, however, may also reflect an inherently different potential of tBid in the process of pore formation. $\mathrm{tBid}$ has been described as a direct activator of pore-forming proteins Bax/Bak. ${ }^{42,43}$ On the contrary, STS-induced MOMP is tBid independent ${ }^{44}$ and dominated by other $\mathrm{BH}$-only proteins that may have different binding characteristics to pro- and antiapoptotic Bcl-2 family members. ${ }^{18,19,42,43}$ This does, however, not exclude that other processes in the upstream signaling pathways during TRAIL- and STS-induced apoptosis may also modulate pore formation kinetics.

In conclusion, the combination of experimental and mathematical systems analyses used in this study provided new functional and mechanistic insight into the spatiotemporal coordination of mitochondrial permeabilization. Our work suggests that the rapidity of MOMP in cells can be sufficiently explained by diffusion-adsorption processes of MOMP inducers and their production kinetics, even if these range from minutes to hours. It also provides the first kinetic description of mitochondrial pore formation within living cells and surprisingly shows that this key process, which is involved in both caspase-dependent and -independent cell death pathways, proceeds with kinetics far more heterogeneously than assumed previously. Furthermore, the approach of rapid imaging and spatial modeling shown here can also be applied to many real-time assays targeting fast and complex intracellular signaling processes.

\section{Materials and Methods}

Materials. Embryo-tested mineral oil and $\mathrm{CHX}$ were obtained from Sigma (Tallaght, Ireland). STS was obtained from Alexis (San Diego, CA, USA) and TRAIL was obtained from Leinco Technologies (St. Louis, MO, USA). TMRM was obtained from MobiTec (Göttingen, Germany). DRB was from Calbiochem (San Diego, CA, USA).
Cell culture. HeLa cells were cultured in RPMI 1640 medium supplemented with penicillin $(100 \mathrm{U} / \mathrm{ml})$, streptomycin $(100 \mu \mathrm{g} / \mathrm{ml})$, and $10 \%$ fetal calf serum (Sigma, Tallaght, Ireland) at $37{ }^{\circ} \mathrm{C}$ and $5 \% \mathrm{CO}_{2}$. Cells were transfected with plasmid DNA (pmyc-CFP-DEVD-YFP or p-cyt-c-EGFP) as described previously. ${ }^{2,9,30}$ Colonies stably expressing the fluorescent probes were isolated following selection with G418 (Invitrogen) for 14 days.

Flow cytometry. After apoptosis induction, HeLa cells were collected with trypsin-EDTA and incubated in binding buffer ( $10 \mathrm{mM}$ HEPES, $135 \mathrm{mM} \mathrm{NaCl}, 5 \mathrm{mM}$ $\mathrm{CaCl}_{2}$ ) containing Annexin-V FITC conjugate $(5 \mu \mathrm{l} / \mathrm{ml}$ ) (BioVision, Mountain View, CA, USA) and propidium iodide (PI) $(1 \mu \mathrm{g} / \mathrm{ml})$ at $37^{\circ} \mathrm{C}$ for $15 \mathrm{~min}$. A total of $10^{5}$ cells were resuspended in ice-cold binding buffer and analyzed on a Cyflow ML 16 flow cytometer (Partec, Münster, Germany). FITC was exited with $10 \%$ of the maximal intensity of the $488 \mathrm{~nm}$ laser and fluorescence emission was collected in the FL1 channel through a $520 \mathrm{~nm}$ band-pass filter; $\mathrm{PI}$ was excited with the $488 \mathrm{~nm}$ laser and fluorescence emission was collected in the FL 3 channel through a $620 \mathrm{~nm}$ long pass filter. A total of $10^{4}$ gated cells were acquired for each sample and analyzed by using Flowmax software (Partec, Münster, Germany).

Time-lapse microscopy of synchrony in apoptosis execution. HeLa cells expressing the DEVDase FRET probe were grown in $\mathrm{CO}_{2}$-equilibrated HEPES-buffered medium (10 mM; pH 7.4) on Willco cover glass dishes (Willco Wells, Amsterdam, Netherlands). Cells were covered with mineral oil and placed in heated $\left(37^{\circ} \mathrm{C}\right)$ incubation chamber mounted on the microscope stage of an epifluorescence microscope (Nikon TE 300), equipped with appropriate polychroic mirror and filter wheels in the excitation and emission light paths. ${ }^{2}$ Cellular proliferation was observed at $\times 4$ or $\times 20$ magnification (Nikon Plan Fluor (NA 0.13) and SFluor (NA 0.75) objectives, respectively) for at least $30 \mathrm{~h}$ and bright-field images were taken at $5 \mathrm{~min}$ intervals. Culture medium was exchanged at a rate of approximately $200 \%$ per $24 \mathrm{~h}$ and allowed for continuous proliferation. Cells were then treated with $1 \mu \mathrm{M}$ STS or $100 \mathrm{ng} / \mathrm{ml}$ TRAIL plus $1 \mu \mathrm{g} / \mathrm{ml} \mathrm{CHX}$, and FRET measurements were performed at 2 min resolution as described previously. ${ }^{9}$ The illumination source was an HBO $103 \mathrm{~W}$ bulb (Osram) and illumination intensity was reduced to approximately $3 \%$ using a sequence of neutral density filters. Depending on $\times 20$ or $\times 4$ magnification and camera binning, illumination duration in the individual channels ranged from 300 to $3000 \mathrm{~ms}$. Images were recorded using a cooled CCD camera (Spot RT SE6, Diagnostic Instruments). The setup was controlled by MetaMorph 7.0 (Universal Imaging, PA, USA). The following parameters were logged for individual cells: time of mother cell division (generation of daughter cells), time of stimulus addition, and time of FRET probe cleavage. Untreated cells served as controls.

Fast sampling of cyt-c-GFP release. Imaging was performed using a Zeiss LSM 510 META inverted confocal microscope (Carl Zeiss, Germany). As for the epifluorescence FRET measurements described above, HeLa cells expressing cyt-c-GFP were incubated onstage over night to allow for sufficient cell division. Using a $\times 63$ oil objective (NA 1.4), GFP was excited with a $488 \mathrm{~nm}$ argon laser line at $1 \%$ output. Fluorescence was collected through a $505-530 \mathrm{~nm}$ band-pass filter; the pinhole was set to $272 \mathrm{~nm}$. Cells were monitored at zoom 0.7 (x,y pixel size $0.2 \mu \mathrm{m}$, pixel dwell time $1.28 \mu \mathrm{s}$ ). Upon drug addition, the full field of view was scanned at $2 \mathrm{~min}$ intervals. Upon apoptotic morphology (cellular shrinkage, blebbing) of an individual cell, the scan area was reduced to include the respective sister cell. Cells were then scanned for 20-30 min in reverse scan mode at intervals of as little as $1 \mathrm{~s}$ (cyt-c-GFP, line step 2). As cellular geometry dictated the scan area, the intervals slightly varied between experiments. Untreated cells served as controls (see also Supplementary Figure 1).

Kinetics of mitochondrial pore formation. The kinetics of pore formation was calculated from the cellular cyt-c-GFP redistribution in cells showing a homogenous onset of cyt-c-GFP release. Cyt-c-GFP redistribution was measured by S.D. of the average pixel intensity of individual cells. ${ }^{4}$ Compartmentalized cyt-c-GFP contributes to a high S.D. and a homogeneous distribution is represented by a low S.D. Changes in S.D. can be related directly to subcellular concentration changes. The asymmetric S.D. traces were fitted with a modified Gompertz function using Origin 7.0 (Northampton, MA, USA):

$$
y=-a * \exp \left(-\exp \left(-k\left(t-t_{c}\right)\right)\right)+b
$$

where $t$ denotes time, $a$ denotes the amplitude, $k$ denotes the decay rate, and $b$ denotes the $y$-offset. $t_{c}$ denotes the time of inflexion, which represents the 
mitochondrial permeabilization allowing for fastest cyt-c release. Onset of pore formation was defined as the time the function deviated by $2 \%$ from the initial baseline. The time from onset to the time of fastest cyt-c release describes mitochondrial pore formation as a measure of time.

Subcellular cyt-c-GFP release waves. The decision as to whether a wave can be observed was drawn visually from analyzing the acquired image sequences, which were recorded at approximately 1 frame per second. The time waves required to fully pass through a cell typically ranged from 30 to $60 \mathrm{~s}$. This analysis therefore was sufficient to unambiguously classify whether cells show a release wave or homogeneous cyt-c release. In cells showing release waves, fluorescent mitochondrial regions were segmented from the cytoplasmic and nucleus regions and the intensity of subcellular regions was plotted against time. Onset of cyt-c-GFP release results in the loss of mitochondrial fluorescence in the respective regions. The average speed of release waves was determined by dividing the distance between the first and last region showing release by the time required to cross this distance. The cross-sectional area of individual cells in confocal scans was determined by generating regions of interest corresponding to the cell shape. Image analysis was performed using LSM Image Examiner (Carl Zeiss, Jena, Germany) and MetaMorph 7.0 (Molecular Devices, UK).

Kymograph analysis. Kymographs showing the change in cyt-c-GFP intensities with time were generated from regions along the cyt-c release wave. Regions were assigned a width of 5 pixels, which, after averaging, represent one line per image frame in the resulting kymograph image. Wave velocities in individual cells were determined in $3 \mu \mathrm{m}$ intervals along the wave front in the kymograph images. Image processing and analysis was performed using MetaMorph 7.0 (Molecular Devices, UK).

FRET disruption kinetics. DEVDase substrate cleavage was detected by FRET analysis. Microscopy images were processed using MetaMorph 7.0 (Molecular Devices, UK) as described previously. 2,10

Statistics. $t$-Test or ANOVA and subsequent Tukey's test were used. Data that were not standard-deviated were analyzed by Mann-Whitney $U$-test. $P$-values below 0.05 were considered to be statistically significant.

Model implementations. Mathematical models were implemented in MATLAB (The Mathworks, UK) for numerical analysis. PDEs were solved using the PDEPE subroutine, which uses an adaptive step Runge-Kutta ordinary differential equation solver (Gear74). Model code is available as Supplementary Information 4 to this article.

Acknowledgements. We thank Aidan Spring for technical support. This research was supported by grants from Science Foundation Ireland (03/RP1/B344; 05/RFP/BIM056), the Health Research Board Ireland (RP/2006/258), the National Biophotonics and Imaging Platform (HEA PRTLI Cycle 4), as well as by SIEMENS Ireland and the EU Framework Programme 7 (APO-SYS).

1. Dussmann H, Rehm M, Kogel D, Prehn JH. Outer mitochondrial membrane permeabilization during apoptosis triggers caspase-independent mitochondrial and caspase-dependent plasma membrane potential depolarization: a single-cell analysis. J Cell Sci 2003; 116: 525-536.

2. Rehm M, Dussmann H, Prehn JH. Real-time single cell analysis of Smac/DIABLO release during apoptosis. J Cell Biol 2003; 162: 1031-1043.

3. Goldstein JC, Munoz-Pinedo C, Ricci JE, Adams SR, Kelekar A, Schuler M et al. Cytochrome $\mathrm{C}$ is released in a single step during apoptosis. Cell Death Differ 2005; 12 : 453-462.

4. Goldstein JC, Waterhouse NJ, Juin P, Evan GI, Green DR. The coordinate release of cytochrome $\mathrm{C}$ during apoptosis is rapid, complete and kinetically invariant. Nat Cell Biol 2000; 2: 156-162.

5. Munoz-Pinedo C, Guio-Carrion A, Goldstein JC, Fitzgerald P, Newmeyer DD, Green DR Different mitochondrial intermembrane space proteins are released during apoptosis in a manner that is coordinately initiated but can vary in duration. Proc Natl Acad Sci USA 2006; 103: 11573-11578.

6. Kumar S. Caspase function in programmed cell death. Cell Death Differ 2007; 14: 32-43.

7. Riedl SJ, Salvesen GS. The apoptosome: signalling platform of cell death. Nat Rev Mol Cell Biol 2007; 8: 405-413.
8. Eckelman BP, Salvesen GS, Scott FL. Human inhibitor of apoptosis proteins: why XIAP is the black sheep of the family. EMBO Rep 2006; 7: 988-994.

9. Rehm M, Dussmann H, Janicke RU, Tavare JM, Kogel D, Prehn JH. Single-cell fluorescence resonance energy transfer analysis demonstrates that caspase activation during apoptosis is a rapid process. Role of caspase-3. J Biol Chem 2002 277: 24506-24514.

10. Rehm M, Huber HJ, Dussmann H, Prehn JH. Systems analysis of effector caspase activation and its control by $\mathrm{X}$-linked inhibitor of apoptosis protein. EMBO J 2006; 25: 4338-4349.

11. Legewie $\mathrm{S}$, Bluthgen $\mathrm{N}$, Herzel $\mathrm{H}$. Mathematical modeling identifies inhibitors of apoptosis as mediators of positive feedback and bistability. PLOS Comput Biol 2006; 2: e120.

12. Luetjens CM, Kogel D, Reimertz C, Dussmann H, Renz A, Schulze-Osthoff K et al. Multiple kinetics of mitochondrial cytochrome $\mathrm{c}$ release in drug-induced apoptosis. Mol Pharmacol 2001; 60: 1008-1019.

13. Wyllie AH, Kerr JF, Currie AR. Cell death: the significance of apoptosis. Int Rev Cytol 1980; 68: 251-306.

14. Messam CA, Pittman RN. Asynchrony and commitment to die during apoptosis. Exp Cell Res 1998; 238: 389-398.

15. Maeder Cl, Hink MA, Kinkhabwala A, Mayr R, Bastiaens PI, Knop M. Spatial regulation of Fus3 MAP kinase activity through a reaction-diffusion mechanism in yeast pheromone signalling. Nat Cell Biol 2007; 9: 1319-1326

16. Markevich NI, Tsyganov MA, Hoek JB, Kholodenko BN. Long-range signaling by phosphoprotein waves arising from bistability in protein kinase cascades. Mol Syst Biol 2006; 2: 61.

17. Pacher P, Hajnoczky G. Propagation of the apoptotic signal by mitochondrial waves. EMBO J 2001; 20: 4107-4121.

18. Zha J, Harada H, Yang E, Jockel J, Korsmeyer SJ. Serine phosphorylation of death agonist BAD in response to survival factor results in binding to 14-3-3 not BCL-X(L). Cell 1996; 87 $619-628$.

19. Akhtar RS, Geng Y, Klocke BJ, Latham CB, Villunger A, Michalak EM et al. BH3-only proapoptotic $\mathrm{Bcl}-2$ family members Noxa and Puma mediate neural precursor cell death. J Neurosci 2006; 26: 7257-7264.

20. Falschlehner $\mathrm{C}$, Emmerich $\mathrm{CH}$, Gerlach B, Walczak $\mathrm{H}$. TRAlL signalling: decisions between life and death. Int J Biochem Cell Biol 2007; 39: 1462-1475.

21. Yamada H, Tada-Oikawa S, Uchida A, Kawanishi S. TRAIL causes cleavage of bid by caspase-8 and loss of mitochondrial membrane potential resulting in apoptosis in BJAB cells. Biochem Biophys Res Commun 1999; 265: 130-133.

22. Hulser DF, Webb DJ. Relation between ionic coupling and morphology of established cells in culture. Exp Cell Res 1973; 80: 210-222.

23. Kumei $Y$, Nakajima T, Sato A, Kamata N, Enomoto S. Reduction of $G 1$ phase duration and enhancement of c-myc gene expression in HeLa cells at hypergravity. J Cell Sci 1989; 93 (Part 2): 221-226

24. Dumitru CA, Gulbins E. TRAIL activates acid sphingomyelinase via a redox mechanism and releases ceramide to trigger apoptosis. Oncogene 2006; 25: 5612-5625.

25. Degli Esposti M, Ferry G, Masdehors P, Boutin JA, Hickman JA, Dive C. Post-translational modification of Bid has differential effects on its susceptibility to cleavage by caspase 8 or caspase 3. J Biol Chem 2003; 278: 15749-15757.

26. Desagher S, Osen-Sand A, Montessuit S, Magnenat E, Vilbois F, Hochmann A et al. Phosphorylation of bid by casein kinases I and II regulates its cleavage by caspase 8 . Mol Cell 2001; 8: 601-611.

27. Sarno S, Reddy H, Meggio F, Ruzzene M, Davies SP, Donella-Deana A et al. Selectivity of 4,5,6,7-tetrabromobenzotriazole, an ATP site-directed inhibitor of protein kinase CK2 ('casein kinase-2'). FEBS Lett 2001; 496: 44-48.

28. Ravi R, Bedi A. Sensitization of tumor cells to Apo2 ligand/TRAlL-induced apoptosis by inhibition of casein kinase II. Cancer Res 2002; 62: 4180-4185

29. Litchfield DW. Protein kinase CK2: structure, regulation and role in cellular decisions of life and death. Biochem J 2003; 369: 1-15.

30. Ward MW, Rehm M, Duessmann H, Kacmar S, Concannon CG, Prehn JH. Real time single cell analysis of Bid cleavage and Bid translocation during caspase-dependent and neuronal caspase-independent apoptosis. J Biol Chem 2006; 281: 5837-5844.

31. Fick A. Über diffusion. Poggendorff's Annalen der Physik und Chemie 1855; 94: 59-86.

32. Luo X, Budihardjo I, Zou H, Slaughter C, Wang X. Bid, a Bcl2 interacting protein, mediates cytochrome $c$ release from mitochondria in response to activation of cell surface death receptors. Cell 1998; 94: 481-490.

33. Farlow SJ. Partial Differential Equations for Engineers and Scientists. John Wiley and Sons: New York, 1993

34. Sigal A, Milo R, Cohen A, Geva-Zatorsky N, Klein Y, Liron Y et al. Variability and memory of protein levels in human cells. Nature 2006; 444: 643-646.

35. Cooper S. Rethinking synchronization of mammalian cells for cell cycle analysis. Cell Mol Life Sci 2003; 60: 1099-1106.

36. Bentele M, Lavrik I, Ulrich M, Stosser S, Heermann DW, Kalthoff $\mathrm{H}$ et al. Mathematical modeling reveals threshold mechanism in CD95-induced apoptosis. J Cell Biol 2004; 166: 839-851.

37. Eissing T, Conzelmann H, Gilles ED, Allgower F, Bullinger E, Scheurich P. Bistability analyses of a caspase activation model for receptor-induced apoptosis. J Biol Chem 2004; 279: 36892-36897.

38. Fussenegger M, Bailey JE, Varner J. A mathematical model of caspase function in apoptosis. Nat Biotechnol 2000; 18: 768-774. 
39. Albeck JG, Burke JM, Aldridge BB, Zhang M, Lauffenburger DA, Sorger PK Quantitative analysis of pathways controlling extrinsic apoptosis in single cells. Mol Cell 2008; 30: 11-25.

40. Huber H, Gomez Estrada G, Dussmann H, O'Connor C, Rehm M. Extending the explanatory power of live cell imaging by computationally modelling the execution of apoptotic cell death. In: Mendez-Vilas A, Diaz J (eds). Formatex Microscopy Book Series: Vol. 1: Badajoz, 2007, pp. 88-99.

41. Hellwig CT, Kohler BF, Lehtivarjo AK, Dussmann H, Courtney MJ, Prehn JH et al. Realtime analysis of TRAIL/CHX-induced caspase activities during apoptosis initiation. J Biol Chem 2008; 283: 21676-21685.
42. Kuwana T, Bouchier-Hayes L, Chipuk JE, Bonzon C, Sullivan BA, Green DR et al. $\mathrm{BH} 3$ domains of $\mathrm{BH} 3-0$ nly proteins differentially regulate Bax-mediated mitochondrial membrane permeabilization both directly and indirectly. Mol Cell 2005; 17: 525-535.

43. Letai A, Bassik MC, Walensky LD, Sorcinelli MD, Weiler S, Korsmeyer SJ. Distinct BH3 domains either sensitize or activate mitochondrial apoptosis, serving as prototype cancer therapeutics. Cancer Cell 2002; 2: 183-192.

44. Kohler B, Anguissola S, Concannon CG, Rehm M, Kogel D, Prehn JH. Bid participates in genotoxic drug-induced apoptosis of HeLa cells and is essential for death receptor ligands' apoptotic and synergistic effects. PLOS ONE 2008; 3: e2844.

Supplementary Information accompanies the paper on Cell Death and Differentiation website (http://www.nature.com/cdd) 